\title{
ROI and Seam-SPIHT based Efficient Image Compression for Mobile Multimedia and Medical Applications
}

\author{
Salija.P \\ Department of ECE \\ Karunya University \\ Coimbatore, India
}

\author{
Manimekalai.M.A.P \\ Department of ECE \\ Karunya University \\ Coimbatore, India
}

\author{
N.A Vasanthi, PhD. \\ Dean \& Head of CSE \\ Nehru institute of Eng\& Tech \\ Coimbatore-105, India
}

\begin{abstract}
There is an increasing demand on content based color image compression in resource limited mobile multimedia devices. Multimedia applications gained popularity in the recent years, but these devices restricted by their display sizes and resource. Most of the devices share the same content on the internet. The size of the display devices is continually changing the quality of the shared content has also get reduced. One solution to this problem is to reduce volume of the multimedia content with less computational complexity to prolong the battery lifetime. The method presented in this paper addresses the increasing demand on content based color image compression and to re target the image at the receiving end with optimum solution.
\end{abstract}

\section{Keywords}

mobile multimedia, DWT, seam carving, SPIHT, color image compression, optimum resolution.

\section{INTRODUCTION}

There are many multimedia devices in the market with different display sizes. They share the same multimedia content on the internet. Also, multimedia applications gained popularity in the last few years. Also, the shared images are color images, content based color image compression is necessary in these devices. Users using different display devices and share the same multimedia content. However, users want to see good quality images in their devices. As the size of the multimedia devices is continually changing the quality of the shared content also gets reduced. These devices have restricted by their display sizes and resources. One solution to this problem is to reduce the volume of the content for the efficient storage space and transmission without losing important information in an image. The existing image compression method which does not support the content based image compression. Most of the compression methods compress the image as a whole. So content based color image compression with less computational complexity and to target the image with optimum resolution at the receiving end is important challenge in the field of resource limited mobile multimedia devices. In this paper, region of interest method used to specify the important region and multilevel decomposition, principle of seam carving also used along with the SPIHT encoder to compress the color image. This technique can also be used in medical images for efficient content based compression.

The main aim of image compression is to reduce the size in bytes to an acceptable level without losing the important information so that more images can stored in the given space or memory location and also reduces the time required to transmit the images over internet or in any other media. Existing image compression method based on discrete wavelet transform [1] (DWT) such as SPIHT [2], JPEG 2000 [3] etc, can meet high quality to the decompressed image. This compresses image as a whole so it does not support content based image compression. Another image compression method is the edge based inpainting [4], in that method some regions of the image skipped from the encoder side and information collected from these regions are delivered to at the encoder side. Same way, data pruning based [5] image compression method compress the image by pruning it to the smaller size before the transmission. These methods do not support content based image compression. Re targeting methods such as in optimized scale and stretch method [6] retargets into different aspect ratios. Image compression based on concentration and dilution [7] use the principle of seam carving [8] for image concentration and interpolation for image re sizing at the receiving end. Seam carving is an image resizing method. Which removes or inserts low energy pixels in an image. In multi-operator media retargeting [9] is used for video re targeting. Quality assessment [10] based image compression method keeps the important region and re sizes the unimportant regions. The aim of the image compression is to reduce the amount of redundancy without losing the important information [11]. In bi-directional similarity [12] method based on re targeting image/video into smaller sizes.

Content based image compression is necessary in the field of mobile multimedia and medical images. The important thing is to define the ROI in an image. There are many image region analysis algorithms. In contrast, based region detection [13] compares the contrast of pixels between nearby region by using salient value. Another method used commonly is the frequency tuned [14], which generate salient map based on the color and luminance. Nowadays there are many images that keeps many features in the same image. So these methods are not suitable for separating ROI and NON-ROI. The method proposed in this paper supports color image compression in resource limited mobile multimedia devices. In the field of medical images, the presented method supports medical image compression and also to preserve important regions in the image. The underlying principle in this content based image compression is the seam carving based SPIHT encoder. At the receiving side user have the greatest choice to re size the image according to the display device without high computational complexity. 


\section{GENERAL DESCRIPTION}

\subsection{SPIHT}

SPIHT [1] (set partitioning in hierarchical trees) is an efficient wavelet based image compression algorithm. It has many advantages over image compression algorithm as compared to other image compression algorithm such as fast encoding/decoding, error protection, high image quality, lossy/lossless compression, progressive image transmission etc., it increases the efficiency of the wavelet transformed images. This image compression method based on the EZW (Embedded Zero tree Wavelet). Also, this method is advantages for the color images. SPIHT makes use of three lists List of Significant Pixels (LSP), List of Insignificant Pixels (LIP) and List of Insignificant Sets (LIS). These are the list of location of coefficients that contain their coordinates. Mainly input images are decomposing into multilevel. SPIHT arranges these wavelets in a tree structure called SOT (Spatial Orientation Tree) which either four child or no children in each level.

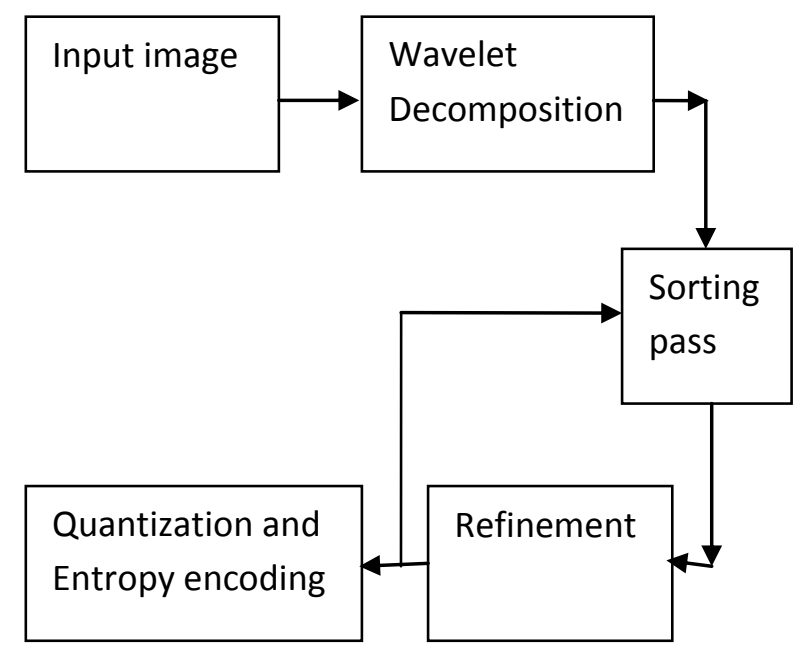

Fig 1: Flow chart of SPIHT

Threshold value of the algorithm can obtained as,

$$
T=\left|\log _{2}\left(\max _{(i, j)}\{c(i, j)\}\right)\right|
$$

This initializes the algorithm, Then performs the steps as shown in the block diagram.

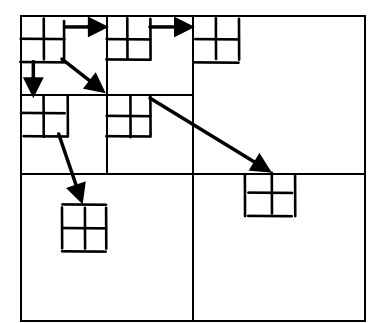

\section{Fig 2:SOT arrangement}

SOT's scanned and encoded in zigzag order. That stars from LSB to MSB. And encoded bits transmitted in a progressive manner.

\subsection{Seam Carving}

Seam carving is an efficient image resizing method. Seam carving [15] is an efficient algorithm for image resizing. Seam is connected path of pixels from top to bottom (vertical) or from left to right (horizontal). Image size can increased by inserting the pixels and reduced by removing the pixels from the image. It produces high quality in re targeted image. Seam carving is mainly for content based image compression. It removes the low energy pixels from an image. Thus compression reduces the consumption of expensive resources.

If $u i$ be the $i$ th part of seam, then $\mathrm{u}_{\mathrm{i}}^{\mathrm{x}}$ and $\mathrm{u}_{\mathrm{i}}^{\mathrm{y}}$ be the vertical and horizontal aspects. The vertical seam is defined as

$$
u^{x}=\left\{u_{i}^{x}\right\}_{i=1}^{N}=\{x(i), i\}_{i=1}^{N}
$$

Where $\mathrm{x}$ is the mapping of $\mathrm{x}:[1, \ldots, N]$, Similarly can write for horizontal seam. It uses an energy function to define the importance of the pixel in an image. Low energy pixels are less significant and removed to reduce the size. Energy function can defined from an image the first step to calculate energy function is to find the gradient image. That can calculate either from luminance channel or from each of the $\mathrm{R}, \mathrm{G}$, and $\mathrm{B}$ channel and taking average of these components. Energy function measures its contrast with its neighbor. From this we need to calculate the energy map to indicate importance of pixel in image that is to be calculated separately for horizontal and vertical seams. suppose $(i, j)$ be the pixel indices in row. The energy map of that pixel be the sum of the minimum values of the neighboring pixels. The pixel indices are shown in the table1.

Table 1. pixel indices

\begin{tabular}{|l|l|l|}
\hline$(i-1, j-1)$ & $(i-1, j)$ & $(i-1, j+1)$ \\
\hline$(i, j-1)$ & $(i, j)$ & $(i, j+1)$ \\
\hline$(i+1, j-1)$ & $(i+1, j)$ & $(i+1, j+1)$ \\
\hline
\end{tabular}

Then we need to remove the low energy pixel from the image. This obtained by finding the low energy pixel from the last row (vertical) or last column (horizontal) and save that location. Then, work backwards to find the minimum of the three neighboring pixels until the last row or column is reached. Then remove these seams and continues this process to remove number of pixels from the image. Optimum seams with minimum energy is found out by using,

$$
u^{*}=\operatorname{argmin} \sum_{i=1}^{J} E\left[I\left(S_{i}\right)\right]
$$

Where E [ ] denotes the energy function. After removing low energy pixels from an image, the pixels must shift either side to maintain the continuity of the image. Cumulative energy cost $d(i, j)$ calculated as,

$d(i, j)=E(i, j)+\min \{d(i-1, j-1), d(I-1, J), M(i-1, j+1)\}$

Energy cost calculates for the three connected path of neighboring pixels. Seam carving is also known as image re targeting algorithm. It functions by preserving the important region and removes the low energy or unimportant regions in an image. Thus it is useful for content based image compression. 


\subsection{ROI and RGB conversion}

In medical images or in the multimedia application we need to keep the important information. Loss of important information is not acceptable. Increasing use of imaging modalities such as Magnetic Resonance Imaging (MRI), Computerized Tomography (CT), Ultrasound (US) etc need the technique to transmit and store the data in an efficient manner. In order to reduce the volume of medical image or multimedia content Region Of Interest (ROI) method is necessary. There are many image images with different features in an image. So ROI extraction is very hard in practical. To increase the robustness we can define the region by our self. So we can preserve the important region of an image. In order to compress color images we need to convert it to $\mathrm{YCbCr}$ format. It is necessary for RGB information encoding. Otherwise, the quality of retargeted images decreases automatically. Y represents the luma component and $\mathrm{Cb}$ and $\mathrm{Cr}$ represents the chroma components. Luma component transmitted with high resolution and high bandwidth and chroma component bandwidth reduced by sub sampled and compressed to improve the system efficiency. RGB to $\mathrm{YCbCr}$ conversion can be achieved by using the equations,

$\mathrm{Y}=(0.257 * \mathrm{R})+(0.504 * \mathrm{G})+(0.098 * \mathrm{~B})+16$

$\mathrm{Cb}=-(0.148 * \mathrm{R})-(0.291 * \mathrm{G})+(0.439 * \mathrm{~B})+12$

$\mathrm{Cr}=(0.439 * \mathrm{R})-(0.368 * \mathrm{G})-(0.071 * \mathrm{~B})+128$

To get back the original $\mathrm{RGB}$ image from the $\mathrm{YCbCr}$ following equations can be used.

$\mathrm{R}=1.164(\mathrm{Y}-16)+1.596(\mathrm{Cr}-128)$

$\mathrm{G}=1.164(\mathrm{Y}-16)-0.813(\mathrm{Cr}-128)-0.391(\mathrm{Cb}-128)$

$\mathrm{B}=1.164(\mathrm{Y}-16)+2.018(\mathrm{Cb}-128)$

The Y component represents the luminance component which depends on the energy of the image in visible band. The $\mathrm{Cb}$, Cr represents. the chrominance components that depend on the wavelength composition and hue and saturation Conversion to $\mathrm{YCbCr}$ allows the more efficient processing and transmission of color componentsRGB images will convert into $\mathrm{YCbCr}$ format for further process.

\section{METHODOLOGY}

An efficient content image compression for both multimedia application and medical image with less computational complexity is an important challenge. We need to re,target the images at the receiving end with optimum resolution. The underlying principle is the ROI based seam SPIHT. Color images are the input, and then convert it to the $\mathrm{YCbCr}$ format for further procedure. Then, we will get three planes. Visual attention analysis is performed to define the important region. So that we can define the region and sharpen that region by using filter. After, this we will get (Region Of Interest) ROI and NON-ROI. The purpose is to give relatively higher weighting factor to ROI and lower value to NON-ROI. Here, we have used the same encoding method for both ROI and NON-ROI. The aim of image compression is to extract the important region which remains unchanged and removes or expands the other region. Analyzed images will decompose into multilevel using 2-d wavelet decomposition. This applies a sequence of low and high pass filter to the input image. Then seam carving is performed to remove the low energy pixels from the image. It removes the unimportant region and preserver the region that we have defined. The number of seams removed from an image depends on the resources in that device and image type. Energy map is generating for the image to indicate the importance. Then SOT (Spatial Orientation Tree) is arranged according to the energy map. SPHT algorithm applied to the carved image and after encoding bit streams are transmitted in a progressive manner.

Block diagram for image encoder and decoder is shown in figure 3 and figure 4 . ROI based seam-SPIHT is the main principle of content based image compression. At the decoder side user has the ultimate choice to re size the image according display size. This technique is applicable for medical images as well.

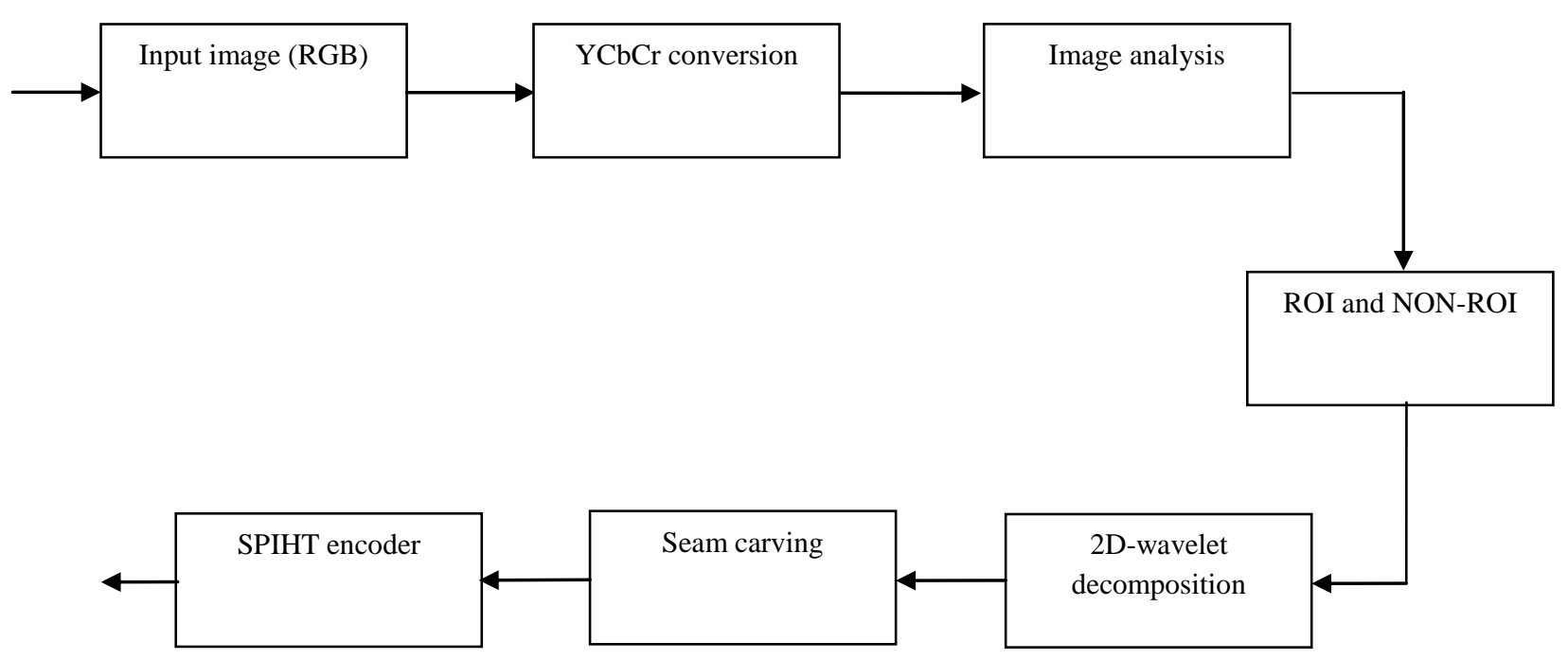

Output

Fig 3: Encoder block diagram 


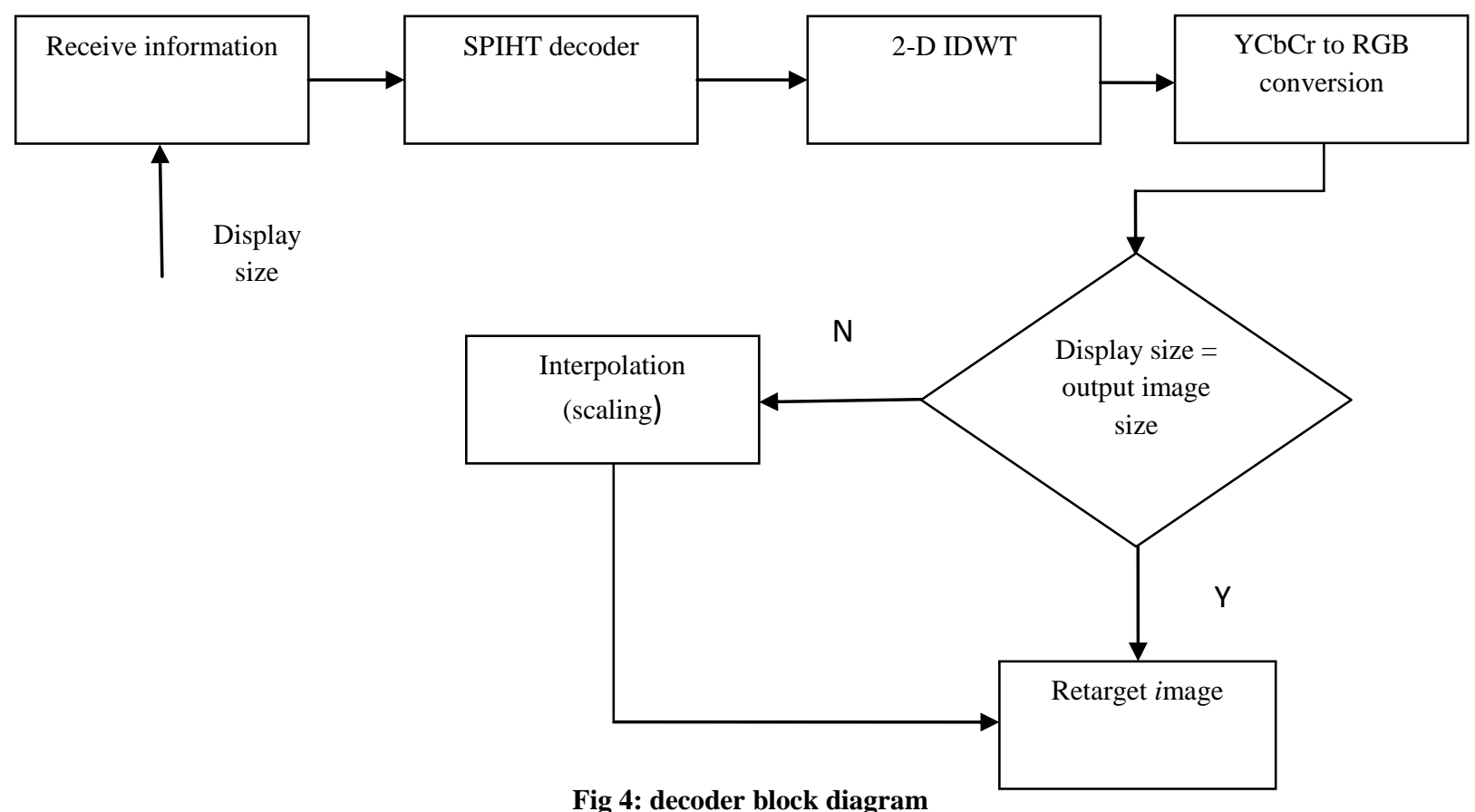

\section{RESULTS AND DISCUSSION}

This section presents the results of experiments conducted to perform content based color image compression with less computational complexity and to re target the image with optimum display size. Experiments conducted in MATLAB. Color Lena image (shown in figure 5) of size $512 \times 512$ is used as input image for conducting the experiments. At the receiver side images reconstructed at the receiver with resolutions $512 \times 512,400 \times 440,410 \times 470$, and $390 \times 460$. The RGB image converted into YCbCr format by using the corresponding equation. ROI extracted to give high weighting factor compared to the rest of the region. Then wavelet decomposition performed on the image to reduce the noise. Carved image after seam carving encoded to transmit efficiently. All the process are applied to three plane is $\mathrm{Y}, \mathrm{Cb}$ and $\mathrm{Cr}$. the carved images are shown in figure 6,7,8. The level of decomposition and the maximum bit rate are determined by the size of image.

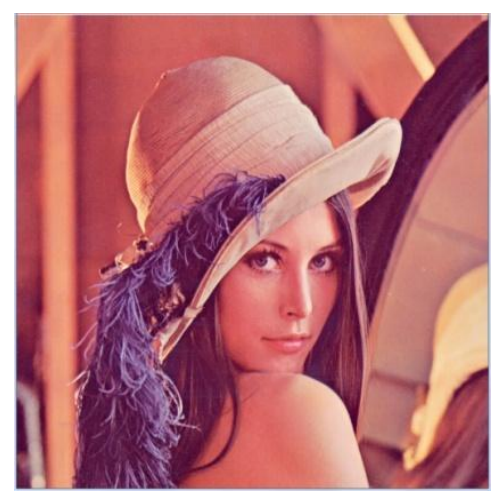

Fig 5: input image

Here sobel operator is used for calculating the gradient of image and vertical seam carving is used. Horizontal seam carving, achieved by rotating the image 180 degree and do the same procedures as that of vertical seam.

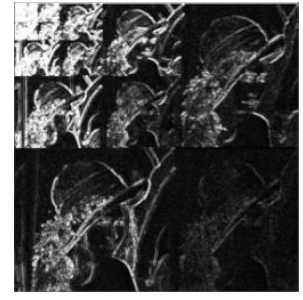

Fig 6:carved image $(\mathbf{Y})$

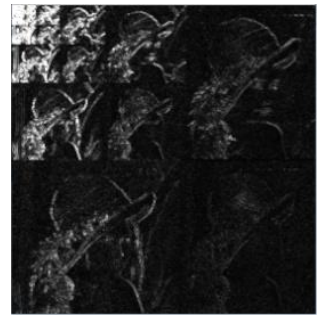

Fig 7:carved image $(\mathrm{Cr})$
During image analysis high contrast is given to the important region in an image.So the seam carving preserves that important region and removes the low energy pixels.

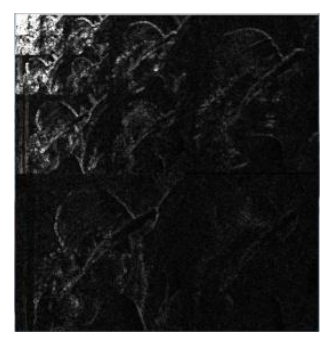

Fig 8. carved image $(\mathrm{Cb})$

Number of level, maximum bits etc, determined from the image itself by using the equation. As we increase the maximum bits and level of decomposition then quality also will increase. Time requirement for a process indicates the computational complexity. User can also define this bit rate and level of decomposition depends on resource and requirement. At the decoder side the user can re size the image according to the display size by using interpolation. The re targetted images are shown in figure9,10,11,12. according to the bitrate different quality is obtained. 


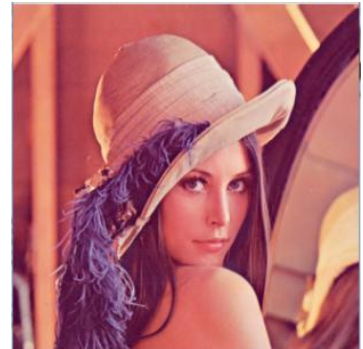

Fig 9: $(512 \times 512)$

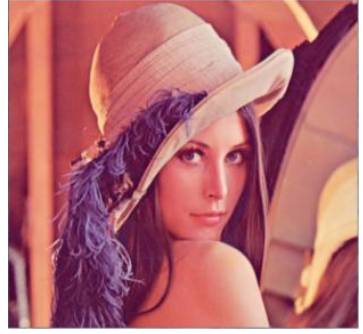

Fig 11: $(410 \times 470)$

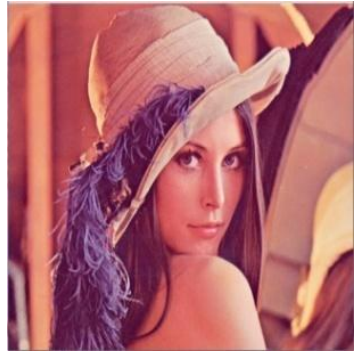

Fig 10: $(400 \times 440)$

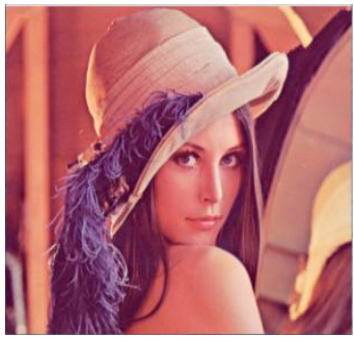

Fig 12; $(390 \times 460)$
Computational complexity at encoder side is much higher than that of spiht alone and computational complexity for different resolution is shown in table 2 .

Table 2 computation time

\begin{tabular}{|l|l|l|l|l|}
\hline \multirow{2}{*}{$\begin{array}{l}\text { Encoder } \\
(\mathrm{s})\end{array}$} & \multicolumn{4}{|l|}{ Decoder (s) } \\
\cline { 2 - 5 } & $\begin{array}{l}512 \times 51 \\
2\end{array}$ & $392 \times 432$ & $412 \times 462$ & $384 \times 486$ \\
\hline 240 & 40.1 & 26.1 & 24.2 & 21.1 \\
\hline
\end{tabular}

The re targeted image maintains quality at the receiver side. The PSNR value obtained for different bitrate are shown in TABLE 3.

Table 3.PSNR value

\begin{tabular}{|c|c|}
\hline Bit rate (bpp) & PSNR (db) \\
\hline 0.25 & 26.63 \\
\hline 0.50 & 29.54 \\
\hline 1.0 & 32.03 \\
\hline 1.5 & 34.16 \\
\hline 2.0 & 35.23 \\
\hline
\end{tabular}

\section{CONCLUSION}

ROI based seam-SPIHT method provides better method for image compression in mobile multimedia applications and medical image compression. For medical images it preserves the important region and removes the unimportant information from the image. In the case of multimedia application content based color image compression achieved with low computational complexity and re targets the images with optimum resolution at the receiving end. ROI's are extracted to give high weighting factor and then 2-D DWT is before seam carving. The SOT's arranged according to the energy map. This indicates the importance of the pixel in image. At the receiving side images reconstructed with optimum resolution. Experimental results show that this method is applicable for both multimedia applications and in the field of medical images since it preserves the important region while compressing and re targets the image with less computational complexity.

\section{REFERENCES}

[1] Abbate A., DeCusatis C. M., and Das P. K., Wavelets and Subbands: Fundamentals and Applications. Boston, MA: Birkhauser, 2002.

[2] [17]Pearlman W. A., and Said A. "A new, fast and efficient image codec based on set partitioning in hierarchical trees," IEEE Trans. Circuits Syst. Video Technol., vol. 6, no. 3, pp. 243-250, Jun. 1996.

[3] Cruz D. S., Ebrahimi T. and Grosbois R., "JPEG 2000 performance evaluation and assessment," Signal Process.: Image Commun., vol. 17, no. 1, pp. 113-130, Jan. 2002.

[4] Liu D., Sun X. Y., Wu F., and Zhang Y. Q. "Image compression with edge-based inpainting," IEEE Trans. Image Process., vol. 17,no. 10, pp. 1273-1287, Oct. 2007.

[5] Nguyen T. Q ., Sole J., Vo D. T., Yin P and Gomila C. "Selective data pruning-based compression using highorder edge-directed interpolation," IEEE Trans. Image Process., vol. 19, no. 2, pp. 399-409, Feb. 2010.

[6] Lee T. Y., Sorkine O., Tai C. L., and Wang Y. S. "Optimized scaleand-stretch for image resizing," ACM Trans. Graphics, vol. 27, no. 5,pp. 118-125, Dec. 2008.

[7] Hasegawa M., Kato S., and Tanaka Y. "Image coding using concentration and dilution based on seam carving with hierarchical search," in Proc. IEEE Int. Conf. Acoust., Speech. Signal Process. Mar. 2010, pp. 1322 1325.

[8] Anh N. T. N., Cai J. F., and Yang W. X., "Seam carving extension: A compression perspective," in Proc. ACM Conf. Multimedia, Oct. 2009,pp. 825-828.

[9] M. Rubinstein, A. Shamir, and S. Avidan, "Multioperator media retargeting,"in Proc. ACM SIGGRAPH, Aug. 2009, pp. 2301-2312.

[10] Chen W. F., Fu X. L., Liu Y. J. and Xuan Y.M., "Image retargeting quality assessment," in Proc., Apr. 2011, vol. 30 , no. 2 , pp. $583-592$.

[11] Cai J. F., Deng C. W., and Lin W. S., "Content-based image compression for arbitrary-resolution display devices,” IEEE Int. 2012.

[12] Caspi, Irani M,Shechtman E. and Simakov D. "Summarizing visual data using bidirectional similarity," in Proc. IEEE Int. Conf. PatternRecognit. Comput. Vis., Jun. 2008, pp. 1-8.

[13] Cheng M. M., Hu S. M., Huang X., Mitra N. J. and Zhang G. X. "Global contrast based salient region detection," in Proc. IEEE Int. Conf. Pattern Recognit. Comput. Vis., Jun. 2011, pp. 409-416.

[14] Achanta.R.,Estrada F., Hemami S., and Susstrunk S., "Frequency-tuned salient region detection," in Proc. IEEE Int. Conf.Pattern Recognit. Comput. Vis., Jun. 2009, pp. 1597-1604.

[15]Avidan S., and Shamir A., "A seam carving for contentaware image resizing,” ACM Trans. , Jul. 2007. 